\section{Stroke outcomes in Thai elderly patients treated with and with- out intravenous thrombolysis}

\author{
Pornpatr A. Dharmasaroja, 1 \\ Sombat Muengtaweepongsa, 1 \\ Junya Pattaraarchachai,2 \\ Permphan Dharmasaroja3
}

1Division of Neurology, Department of Internal Medicine, Faculty of Medicine, Thammasat University, Pathumthani; 2Data Analysis and Management Unit, Department of Community Medicine, Faculty of Medicine, Thammasat University, Pathumthani; ${ }^{3}$ Department of Anatomy, Faculty of Science, Mahidol University, Bangkok, Thailand

\section{Abstract}

Higher mortality was found in very old patients with acute ischemic stroke treated with intravenous recombinant tissue-plasminogen activator (rtPA) as compared to younger patients. The benefit of thrombolytic treatment in this particular subgroup is still a subject of debate. The purpose of this study was to compare stroke outcomes in Thai patients aged over 70 years treated with and without intravenous rtPA. This was a retrospective review of sequential cases and was not a randomized controlled study. One-hundred and five patients with acute ischemic stroke aged over 70 years who were treated with intravenous rtPA and 105 patients without rtPA treatment (control group) were included in the study. Patients' base-line characteristics and study outcomes of interest were compared. There were significant differences in the base-line characteristics of the two groups. However, for the subgroup of patients aged over 80 years, these characteristics were similar. Those who were treated with intravenous rtPA had a higher rate of favorable outcomes $(40 \% v s$ $16 \% ; \mathrm{P}=0.137$ ) and a lower rate of mortality ( $22 \%$ vs $44 \% ; \mathrm{P}=0.128$ ) than patients who did not receive rtPA treatment. In well-matched subgroups of patients aged over 80 years, our retrospective review revealed there was a trend of a higher rate of favorable outcome and lower mortality in patients receiving rtPA treatment. More study is needed to further confirm the suggested benefit of thrombolysis in Asian octogenarian acute stroke patients.

\section{Introduction}

Although intravenous thrombolysis has become a standard treatment for acute ischemic stroke in eligible patients, the recommendations for treating patients aged over 80 years are still a subject of debate. Many studies have revealed a higher mortality rate and lower favorable outcome rate in patients aged over 80 years. In some studies, the rate of symptomatic intracerebral hemorrhage (ICH) was higher in patients aged over 80 years while in others it was similar to that observed in younger patients. ${ }^{1-6}$ However, a large study and a meta-analysis have both recently shown that older patients benefit from treatment at least as much as younger patients. ${ }^{7,8}$

Most of the data are from Western countries. Menon et al. assessed risk score for intracranial hemorrhage after intravenous thrombolytic treatment in 10,242 patients with acute ischemic stroke and found that Asian race was one of the independent risk predictors. ${ }^{9}$ Data in Asian patients may be different from that in Western populations. Thrombolytic studies from some Asian countries, such as Taiwan and Vietnam, have not included patients aged over 80 years. ${ }^{10,11}$ Previous studies in Thai patients showed that older age ( $\geq 70$ years old) was inversely associated with early improvement. Significantly higher rates of mortality and symptomatic ICH were also found in patients aged 70 years or over as compared with younger patients after treatment with recombinant tissue-plasminogen activator (rtPA). ${ }^{12,13}$ However, whether treatment with thrombolysis will have a greater beneficial effect on outcomes than treatment without rtPA in Thai elderly patients is unknown. The purpose of this study was to compare stroke outcomes in patients aged over 70 years treated with and without intravenous thrombolysis.

\section{Materials and Methods}

This was a case-control study. The number of patients on each arm was calculated by the following formula (equal to 82):

$$
\frac{\left(\mathrm{Z}_{1-\alpha} \sqrt{2 \mathrm{P}(1-\mathrm{P})}+\mathrm{Z}_{1-\beta} \sqrt{\mathrm{P}_{1}\left(1-\mathrm{P}_{1}\right)+\mathrm{P}_{0}\left(1-\mathrm{P}_{0}\right)}\right)^{2}}{\left(\mathrm{P}_{1}-\mathrm{P}_{0}\right)^{2}}
$$

where:

$\mathrm{P} 1=0.40, \mathrm{P} 0=0.20$, power of the test $80 \%$, confidence level $95 \%$.

One-hundred and five patients aged over 70 years with acute ischemic stroke treated with intravenous rtPA at Thammasat University Hospital, Thailand, between June 2007 and January 2011 were identified from the Thammasat Stroke Registry and were included in the study. One-hundred and five patients aged over 70 years who were seen from May 2010 to January 2011, and who were not treat-
Correspondence: Pornpatr A. Dharmasaroja, Division of Neurology, Faculty of Medicine Thammasat University, Klong 1, Klong Luang, Pathumthani 12120, Thailand.

Tel. +66.865.881734 - Fax: +66.292.69793

E-mail: pornpatr1@hotmail.com

Key words: thrombolysis, stroke, aging, Asian, Thai.

Contributions: PAD, study design, data collection, data analysis and writing the manuscript; SM, some data collection; JP, data analysis; PD, study design, data analysis.

Conflict of interests: the authors declare no potential conflict of interests.

Received for publication: 22 October 2012.

Revision received: 26 March 2013.

Accepted for publication: 8 April 2013.

This work is licensed under a Creative Commons Attribution NonCommercial 3.0 License (CC BYNC 3.0).

() Copyright P.A. Dharmasaroya et al., 2013

Licensee PAGEPress, Italy

Neurology International 2013; 5:e15

doi:10.4081/ni.2013.e15

ed with intravenous thrombolysis, were included as control.

Intravenous rtPA was prescribed for acute ischemic stroke patients within $3 \mathrm{~h}$ of onset if there were no contraindications. However, after the publication of the European Cooperative Acute Stroke Study III (ECASS III), and the recommendations with regard to widening the time window for the treatment of acute ischemic stroke with intravenous rtPA issued by the American Heart Association/ American Stroke Association, 14,15 we extended the time window for treatment with rtPA to 4.5 h. Most contraindications were the same as in the guidelines for treatment of acute ischemic stroke from the American Heart Association/ American Stroke Association. ${ }^{14}$ However, older age ( $>80$ years old) was not an exclusion criterion. Patients with high blood pressure (systolic blood pressure $>185 \mathrm{mmHg}$ or diastolic blood pressure $>110 \mathrm{mmHg}$ ) were not excluded as long as blood pressure could be controlled by intravenous nicardipine (as defined by achieving target systolic blood pressure $<185 \mathrm{mmHg}$ and diastolic blood pressure $<110$ $\mathrm{mmHg}$ ) before administration of rtPA. Abnormal findings from brain computed tomography (CT) including: i) hyperdense lesions suggestive of bleeding; and ii) hypodense lesions accounting for more than onethird of the middle cerebral artery (MCA) distribution were also contraindications for thrombolysis. Patients who were treated with 
intravenous $\mathrm{rtPA}(0.9 \mathrm{mg} / \mathrm{kg})$ were admitted to an intensive care unit or stroke unit to monitor and control blood pressure with a clinical follow up for the first $24 \mathrm{~h}$. The severity of the stroke was evaluated using the National Institutes of Health Stroke Scale (NIHSS) by the same doctors before and after receiving rtPA. Another CT brain scan was performed within $36 \mathrm{~h}$ in all patients who received rtPA. However, follow-up brain CT was not routinely performed in all of those patients who were not treated with intravenous thrombolysis. The modified Rankin scale (mRS) was used to assess outcomes of the patients three months after the stroke onset. Symptomatic ICH was also classified by the National Institute of Neurological Disorders (NINDS) stroke rtPA criterion, i.e. hemorrhage associated with worsening of 1 point or more in the NIHSS score. 16 Early improvement was defined as having complete recovery or marked clinical improvement (decrease in NIHSS from baseline $>8$ points) $24 \mathrm{~h}$ after admission.

The base-line characteristics of patients, including age, gender, cardiovascular risk factors, blood glucose at presentation, platelet count, prothrombin time, severity of stroke, and stroke subtypes were studied. The measured outcome variables of this study were early improvement at $24 \mathrm{~h}$, symptomatic intracerebral hemorrhage, favorable outcome (mRS 0.1 ) and death at three months. Patients' demographics, vascular risk factors and measured outcome variables were compared between those who were treated with and those without intravenous thrombolysis; an independent-samples t-test for continuous variables and the $\chi^{2}$ test for dichotomous variables were used. The data are presented as means for continuous variables and percentage (number) for dichotomous variables. This study was approved by the human ethics committee of the Faculty of Medicine, Thammasat University, Thailand.

\section{Results}

One-hundred and five patients aged over 70 years were not treated with intravenous rtPA during the study period; of these, 49 patients presented within $4.5 \mathrm{~h}$ after stroke onset. The reasons for not prescribing rtPA were: delays in the intervention process, i.e. late presentation, delay in laboratory or CT process $(n=14$, 28.6\%); excessively mild stroke severity, i.e. NIHSS under $4(n=12,24.5 \%)$; rapid improvement $(n=5,10.2 \%)$; abnormal CT findings i.e. hypodense lesion over one-third of the MCA distribution ( $\mathrm{n}=16,32.7 \%)$; recent major surgery $(n=1,2 \%)$; and history of intracerebral hemorrhage, ICH $(n=1,2 \%)$. Follow-up CT or magnetic resonance imaging (MRI) was per- formed in 48 patients who did not receive rtPA. The base-line characteristics of the patients with and those without rtPA treatment are presented in Table 1. There were significant differences in base-line characteristics associated with older age (78 vs $76 ; \mathrm{P}=0.03$ ), milder stroke (10 vs $13 ; \mathrm{P}=0.001)$, lower blood sugar at presentation (116 vs $135 ; \mathrm{P}=0.026)$ and reduced frequency of cardioembolic stroke ( $25 \%$ vs $42 \% ; \mathrm{P}=0.009$ ) in patients without the rtPA treatment as compared with those with the rtPA treatment. However, there was no significant difference in the rates of favorable outcomes $(41 \%$ vs $37 \% ; \mathrm{P}=0.56)$ or mortality ( $25 \%$ vs $22 \% ; \mathrm{P}=0.69)$ in association with a marginally higher rate of symptomatic intracerebral hemorrhage ( $4 \%$ vs $14 \% ; \mathrm{P}=0.09$ ) in those receiving rtPA treatment. A significantly higher rate of early improvement at $24 \mathrm{~h}$ was found in patients treated with rtPA (4\% vs $23 \%$; $\mathrm{P}=0.001)$. Univariate analysis and multiple logistical regression analysis were carried out to look for the factors associated with early improvement. Only rtPA treatment was related to early improvement (OR 14.1, 95\% CI: 1.6124.7) after adjustment for gender, stroke subtypes, hyperlipidemia, coronary artery disease, atrial fibrillation, antithrombotic treatment and symptomatic intracerebral hemorrhage. Stroke outcomes at three months as measured by the modified Rankin Scale (mRS) are presented in Figure 1.

There were significant differences in some base-line characteristics in the subgroup of patients aged over 80 years, with a greater history of hypertension and higher mean age, milder stroke, and a reduced risk of cardioembolic stroke in patients not receiving rtPA treatment (Table 1). Patients ( $>80$ years old) treated with intravenous rtPA had a higher rate of favorable outcomes (40\% vs $16 \%$; $\mathrm{P}=0.137)$ and a lower rate of mortality ( $22 \%$ vs $44 \% ; \mathrm{P}=0.128$ ) than patients not receiving rtPA treatment.

\section{Discussion}

The number of elderly patients is increasing in most countries and consequently strokes in the very old ( $>80$ years) subgroup occur more often; approximately one-third of all stroke patients are in this subgroup. ${ }^{17,18}$ Mortality rates and non-dependency were higher in patients aged over 80 years as compared with the younger patients. Higher 1-month mortality rates were $31-34.6 \%$ in the very old patients (>80 years) as compared with the younger patients (13.4-16.7\%); one study reported a 1year mortality rate of $51.6 \% .^{19,20}$ Disability after stroke ( $\mathrm{mRS} \geq 3$ ) was reported in $78 \%$ of the very old patients who survived. ${ }^{20}$ Our study showed that the 3-month mortality rate was $44 \%$ in patients aged over 80 years who did not receive rtPA treatment. Causes of high mortality may result from the reduced utilization of health care resources or the comorbidity and high proportion of atrial fibrillation reported in association with stroke.18,19,21 Comorbidity, cognitive impairment, marital status, absence of caregivers and a lack of motivation that reduced the effectiveness of rehabilitation programs were potential contributors to the high level of disability observed in stroke survivors. ${ }^{22-25}$

Thrombolytic treatment in very old patients with acute ischemic stroke is still a subject of debate. The National Institute of Neurological Disorders and Stroke (NINDS) included all age ranges of the patients whereas the European Cooperative Acute Stroke Study (ECASS) excluded patients aged over 80 years. ${ }^{14,16}$ Thus, the European Medicine Evaluation Agency has not approved thrombolysis with alteplase for use in patients aged over 80 years. ${ }^{26} \mathrm{~A}$ systemic review and meta-analysis included 13 studies comparing outcomes after intravenous thrombolysis among 764 elderly patients (age $\geq 80$ years) and 2792 patients under 80 years of

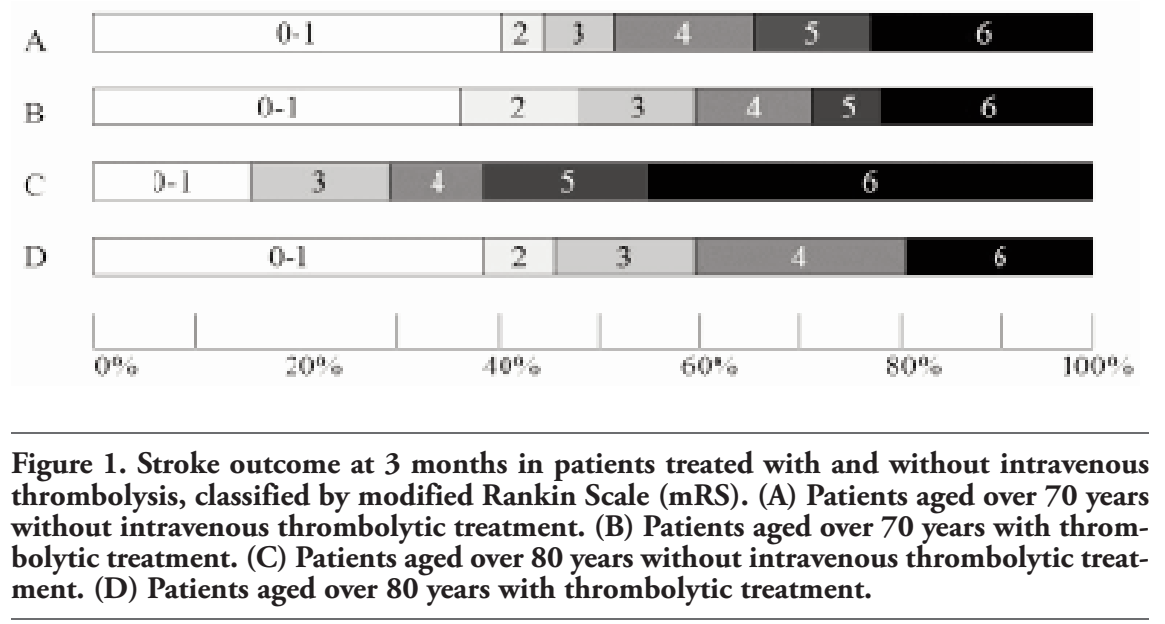


age. Elderly patients achieved less favorable outcomes (OR 0.49, 95\% CI: 0.40-0.61) and showed higher mortality rates (OR 2.77, 95\% CI: 2.25-3.40) but not significantly higher symptomatic ICH rates (OR 1.31, 95\% CI: 0.931.84 ) as compared with patients under 80 years old. ${ }^{27}$ Ford et al. studied outcomes and symptomatic ICH rates in 19,411 patients aged 80 years or under and 1831 patients aged over 80 years in the Safe Implementation of Treatment in Stroke - International Stroke Thrombolysis Register (SITS-ISTR). ${ }^{3}$ Here patients over 80 years of age had a higher mortality rate $(30 \%$ vs $12 \%$, OR $1.53,95 \%$ CI: 1.43 $1.65)$ and were less independent (35\% vs $57 \%$, OR 0.73, 95\% CI: 0.68-0.78). There was a nonsignificant increase in the symptomatic ICH rate according to NINDS criteria in the older subgroup ( $9.5 \%$ vs $7.8 \%$, OR $0.96,95 \%$ CI: 0.87 1.06). Mishra et al. assessed the effect of age in response to alteplase by comparing 23,334 patients who received thrombolysis from the SITS-ISTR (during 2002-2009) with a control group made up of 6166 patients who participated in VISTA neuroprotection trials (during 1998-2007) and were not treated with rtPA.28 Among the subgroup of patients aged over 80 years, 2235 and 1237 patients were treated with and without rtPA, respectively. As compared with a control subgroup, very old patients ( $>80$ years) treated with rtPA had a higher rate of favorable outcomes (mRS 0-2) (OR 2.1, 95\% CI: 1.7-2.5) and a marginally lower mortality rate (OR $0.89,95 \%$ CI: 0.76 1.0). The third international stroke trial (IST3) compared the efficacy and safety of rtPA $v s$ placebo, with a sample size of 3035 patients and including 1617 patients aged over 80 years. ${ }^{7}$ In a subgroup of patients aged over 80 years, the rate of favorable outcome was higher in those receiving rtPA treatment $(27.3 \%$ vs $23.5 \%$, OR 1.35, 95\% CI: 0.97-1.88) and this did not differ significantly from the younger patients.Our study showed non-significant differences in the rates of favorable outcomes, symptomatic intracerebral hemorrhages and mortality in patients aged over 70 years with or without thrombolytic treatment. However, there were significant differences in several base-line characteristics between the groups. Older age, severe stroke, high blood glucose, cardioembolic stroke had been reported to be the independent factors of poor outcomes and death. Thus, it is not possible to conclude that

Table 1. Base-line characteristics and outcomes in aging patients ( $>70$ years old) treated with and without intravenous recombinant-tissue plasminogen activator.

\begin{tabular}{|c|c|c|c|c|c|c|}
\hline Baseline characteristics & No rtPA $(\mathrm{N}=105)$ & $\begin{array}{l}>70 \text { years old } \\
\text { rtPA }(\mathrm{N}=105)\end{array}$ & $\mathbf{P}$ & No rtPA $(\mathrm{N}=32)$ & $\begin{array}{l}>80 \text { years old } \\
\text { rtPA }(\mathrm{N}=18)\end{array}$ & $\mathbf{P}$ \\
\hline Mean age (years) (median, range) & $78 \pm 6(76,71-94)$ & $76 \pm 5(75,71-92)$ & 0.033 & $87 \pm 4$ & $85 \pm 4$ & 0.325 \\
\hline Mean NIHSS & $10 \pm 7$ & $13 \pm 7$ & 0.001 & $11 \pm 7$ & $15 \pm 9$ & 0.188 \\
\hline Mean NIHSS at $24 \mathrm{~h}$ & $10 \pm 8$ & $10 \pm 8$ & 0.784 & $11 \pm 7$ & $11 \pm 9$ & 0.926 \\
\hline Mean prothrombin time (seconds) & $13.4 \pm 10.3$ & $12.2 \pm 2.1$ & 0.263 & $15.7 \pm 14$ & $12.7 \pm 1.5$ & 0.391 \\
\hline Mean INR & $1.05 \pm 0.15$ & $1.04 \pm 0.16$ & 0.919 & $1.03 \pm 0.95$ & $1.09 \pm 0.2$ & 0.317 \\
\hline Mean platelet count $(\times 109 / \mathrm{L})$ & $228 \pm 74$ & $238 \pm 81$ & 0.419 & $222 \pm 59$ & $230 \pm 59$ & 0.662 \\
\hline Mean LDL (mg/dL) & $122 \pm 44$ & $116 \pm 36$ & 0.270 & $121 \pm 34$ & $117 \pm 37$ & 0.703 \\
\hline Mean blood glucose at presentation (mg/dL) & $116 \pm 49$ & $135 \pm 70$ & 0.026 & $116 \pm 43$ & $136 \pm 74$ & 0.322 \\
\hline $\begin{array}{l}\text { Gender } \\
\text { Male } \\
\text { Female }\end{array}$ & $\begin{array}{l}57(54 \%) \\
48(46 \%)\end{array}$ & $\begin{array}{l}55(52 \%) \\
50(48 \%)\end{array}$ & 0.782 & $\begin{array}{l}11(34 \%) \\
21(66 \%)\end{array}$ & $\begin{array}{l}8(44 \%) \\
10(56 \%)\end{array}$ & 0.481 \\
\hline $\begin{array}{l}\text { NIHSS at presentation } \\
0-5 \\
6-10 \\
11-15 \\
>15\end{array}$ & $\begin{array}{l}39(37 \%) \\
24(23 \%) \\
17(16 \%) \\
25(24 \%)\end{array}$ & $\begin{array}{l}14(13 \%) \\
30(29 \%) \\
18(17 \%) \\
43(41 \%)\end{array}$ & 0.001 & $\begin{array}{c}7(22 \%) \\
10(31 \%) \\
6(19 \%) \\
9(28 \%)\end{array}$ & $\begin{array}{l}2(11 \%) \\
5(28 \%) \\
2(11 \%) \\
9(50 \%)\end{array}$ & 0.434 \\
\hline Hypertension & $71(68 \%)$ & $74(71 \%)$ & 0.654 & $23(72 \%)$ & $7(39 \%)$ & 0.022 \\
\hline Diabetes mellitus & $24(23 \%)$ & $19(18 \%)$ & 0.939 & $8(25 \%)$ & $3(17 \%)$ & 0.724 \\
\hline Hyperlipidemia & $30(29 \%)$ & $30(29 \%)$ & 1.000 & $7(22 \%)$ & $3(17 \%)$ & 0.730 \\
\hline Coronary artery disease & $14(13 \%)$ & $16(15 \%)$ & 0.693 & $4(13 \%)$ & $3(17 \%)$ & 0.692 \\
\hline Old ischemic stroke & $4(4 \%)$ & $10(10 \%)$ & 0.093 & $1(3 \%)$ & $2(11 \%)$ & 0.291 \\
\hline Transient ischemic attack & $2(2 \%)$ & $4(4 \%)$ & 0.683 & $1(3 \%)$ & - & - \\
\hline Atrial fibrillation & $29(28 \%)$ & $36(34 \%)$ & 0.296 & $12(38 \%)$ & $5(28 \%)$ & 0.486 \\
\hline Smoking & $15(14 \%)$ & $20(19 \%)$ & 0.355 & $4(13 \%)$ & $5(28 \%)$ & 0.253 \\
\hline Antithrombotic use & $36(34 \%)$ & $37(35 \%)$ & 0.847 & $10(32 \%)$ & $3(17 \%)$ & 0.315 \\
\hline $\begin{array}{l}\text { Stroke subtype } \\
\text { LAA } \\
\text { SAO } \\
\text { CE } \\
\text { UND }\end{array}$ & $\begin{array}{l}31(30 \%) \\
37(35 \%) \\
26(25 \%) \\
11(10 \%)\end{array}$ & $\begin{array}{c}33(31 \%) \\
26(25 \%) \\
44(42 \%) \\
2(2 \%)\end{array}$ & 0.009 & $\begin{array}{c}7(22 \%) \\
13(41 \%) \\
9(28 \%) \\
3(9 \%)\end{array}$ & $\begin{array}{l}7(39 \%) \\
3(17 \%) \\
8(44 \%) \\
-\end{array}$ & 0.144 \\
\hline Early improvement at $24 \mathrm{~h}$ & $4(4 \%)$ & $24(23 \%)$ & 0.001 & - & $4(22 \%)$ & 0.058 \\
\hline Asymptomatic intracerebral hemorrhage & $11(23 \%)$ & $32(31 \%)$ & 0.318 & $2(14 \%)$ & $3(17 \%)$ & 1.000 \\
\hline Symptomatic intracerebral hemorrhage & $2(4 \%)$ & $14(14 \%)$ & 0.095 & $1(7 \%)$ & - & 0.438 \\
\hline Favorable outcome at 3 months & $43(41 \%)$ & $39(37 \%)$ & 0.557 & $5(16 \%)$ & $7(40 \%)$ & 0.137 \\
\hline Mortality rate at 3 months & $26(25 \%)$ & $23(22 \%)$ & 0.690 & $14(44 \%)$ & $4(22 \%)$ & 0.128 \\
\hline
\end{tabular}

rtPA, recombinant tissue-plasminogen activator; NIHSS, National Institutes of Health Stroke Scale. 
thrombolytic treatment is ineffective in aging patients. Differences in favorable outcomes and death between those with and without rtPA treatment were obvious in subgroups of patients aged over 80 years. Patients with the rtPA treatment had a higher rate of favorable outcomes ( $40 \%$ vs $16 \% ; \mathrm{P}=0.137)$ and a lower rate of mortality ( $22 \%$ vs $44 \%$; $\mathrm{P}=0.128$ ) compared with those without the rtPA treatment. However, the difference did not reach statistical significance, which may largely be due to the small number of patients in this subgroup.

The mechanism of early recovery or improvement is not clearly understood. A transcranial Doppler (TCD) study in patients treated with rtPA found that the timing of arterial recanalization, as determined by TCD, correlated with early clinical recovery from stroke. ${ }^{29}$ Several studies reported a similar proportion of successful recanalization in patients aged over 80 years to that achieved in younger patients. ${ }^{3}$ Our study showed that early improvement within $24 \mathrm{~h}$ after stroke onset was found more frequently in patients who received the rtPA treatment, which may represent a higher rate of recanalization.

\section{Conclusions}

Our study has some limitations. Firstly, this was a retrospective review of sequential cases and was not a randomized controlled study. Non-thrombolytic patients ( $>70$ years of age) were classified by the duration of time required to reach similar numbers to those achieved in patients treated with rtPA. Significant differences in base-line characteristics of the two groups were noted. The differences in stroke severity between the case and control groups may represent more rapid stroke awareness in patients with more severe stroke, as this subgroup of patients was more likely to receive thrombolytic treatment. However, for the subgroup of the patients aged over 80 years, characteristics were fairly similar. Second, a follow-up brain CT or MRI was not performed in some of the patients who did not receive rtPA treatment; therefore, the rate of asymptomatic intracerebral hemorrhage may be underestimated.

This is the first study in Thailand comparing stroke outcomes in aging patients with and without rtPA treatment. The study showed that the very old patients receiving rtPA did not have poorer outcomes. Further randomized controlled studies are still needed to confirm the suggested benefit of thrombolysis in aging Asian patients.

In conclusion, in subgroups of patients aged over 80 years, this retrospective review revealed that there was a trend of higher rate of favorable outcome and lower mortality in patients receiving rtPA treatment.

\section{References}

1. Engelter ST, Bonati LH, Lyer PA. Intravenous thrombolysis in stroke patients of $\geq 80$ versus $<80$ years of age a systemic review across cohort studies. Age Ageing 2006;35:572-80.

2. Forster A, Szabo K, Kreisel S, et al. Thrombolysis in very old people with stroke: stroke subtypes, patterns, complications, and clinical outcome. J Am Geriatr Soc 2011;59:178-80.

3. Ford GA, Ahmed N, Azevedo E, et al. Intravenous alteplase for stroke in those older than 80 years old. Stroke 2010;41: 2568-74.

4. Toni D, Lorenzano S, Agnelli G, et al. Intravenous thrombolysis with rt-PA in acute ischemic stroke patients aged older than 80 years in Italy. Cerebrovasc Dis 2008;25:129-35.

5. Costello CA, Campbell BCV, Perez de la Ossa N, et al. Age over 80 years is not associated with increased hemorrhagic transformation after stroke thrombolysis. J Clin Neurosci 2012;19:360-3.

6. Breure L, Blinzler C, Huttner HB, et al. Offlabel thrombolysis for acute ischemic stroke: rate, clinical outcome and safety are influenced by the definition of minor stroke. Cerebrovasc Dis 2011;32:177-85.

7. The IST-3 collaborative group. The benefits and harms of intravenous thrombolysis with recombinant tissue plasminogen activator within $6 \mathrm{~h}$ of acute ischaemic stroke (the third international stroke trial [IST-3]: a randomized controlled trial. Lancet 2012;379:2352-63.

8. Wardlaw JM, Murray V, Berge E, et al. Recombinant tissue plasminogen activator for acute ischaemic stroke: an updated systemic review and meta-analysis. Lancet 2012;379:2364-72.

9. Menon BK, Saver JL, Prabhakaran S, et al. Risk score for intracranial hemorrhage in patients with acute ischemic stroke treated with intravenous tissue-type plasminogen activator. Stroke 2012;43:2293-9.

10. Chao AC. Thrombolysis for acute ischemic stroke in Taiwan. Acta Neurol Taiwan 2010;19:225-7.

11. Nguyen $\mathrm{TH}$, Troung AL, Ngo AB, et al. Patients with thrombolysed stroke in Vietnam have an excellent outcome: results from the Vietnam Thrombolysis Registry. Eur J Neurol 2010;17:1188-92.

12. Dharmasaroja PA, Muengtaweepongsa S, Dharmasaroja P. Early outcome after intravenous thrombolysis in patients with acute ischemic stroke. Neurology India 2011;59:351-4.

13. Dharmasaroja PA, Muengtaweepongsa S, Dharmasaroja P. Intravenous thrombolysis in Thai patients with acute ischemic stroke: role of aging. J Stroke Cerebrovasc Dis 2013;22:227-31.

14. Hacke W, Kaste M, Bluhmki E, et al. Thrombolysis with alteplase 3 to 4.5 hours after acute ischemic stroke. N Engl J Med 2008;359:1317-29.

15. Del Zoppo GL, Saver JL, Jauch EC, et al. Expansion of the time window for treatment of acute ischemic stroke with intravenous tissue plasminogen activator: a science advisory from the American heart association/American stroke association. Stroke 2009;40:2945-8.

16. The National Institute of Neurological Disorders and Stroke rt-PA Stroke Study Group. Tissue plasminogen activator for acute ischemic stroke. N Engl J Med 1995;333:1581-7.

17. Russo T, Felzani G, Marini C. Stroke in the very old: a systemic review of studies on incidence, outcome, and resource use. $\mathrm{J}$ Aging Res 2011;2011:108785.

18. Marini C, Baldassarre M, Russo T, et al. Burden of first-ever ischemic stroke in the oldest old. Neurology 2004;62:77-81.

19. Palnum KD, Petersen P, Sorensen HT, et al. Older patients with acute stroke in Denmark: quality of care and short-term mortality. A nationwide follow-up study. Age Ageing 2008;37:90-5.

20. Olindo S, Cabre P, Deschamps R, et al. Acute stroke in the very elderly. Epidemilogical features, stroke subtypes, management and outcome in Martinique, French West Indies. Stroke 2003;34:1593-7.

21. Lee M, Ovbiagele B. Navigating the gray zones of stroke. Management for a graying population. Cerebrovasc Dis 2010;29:523-7.

22. Carlo AD, Lamassa M, Pracucci G, et al. Stroke in the very old. Clinical presentation and determinants of 3-month functional outcome: a European perspective. Stroke 1999;31:2313-9.

23. Rockwood K, Stolee P, McDowell I. Factors associated with institutionalization of older people in Canada: Testing a multifactorial definition of frailty. J Am Geriatr Soc 1996;44:578-82.

24. Mysiw WJ, Beegan JG, Gatens PF. Prospective cognitive assessment of stroke patients before inpatient rehabilitation: the relationship of the neurobehavioral cognitive status examination to functional improvement. Am J Phys Med Rehabil 1989;68:168-71.

25. Falconer JA, Naughton BJ, Strasser DC, Sinacore JM. Stroke inpatient rehabilitation: a comparison across age groups. J Am Geriatr Soc 1994;42:39-44.

26. Lees KR, Bluhmki E, von Kummer R, et al. Time to treatment with intravenous alteplase and outcome in stroke: an updated pooled analysis of ECASS, ATLANTIS, NINDS and EPITHET trials. Lancet 2010;375:1695-703. 
27. Bhatnagar P, Sinha D, Parker RA, et al. 28. Mishra NK, Ahmed N, Anderson G, et al. Intravenous thrombolysis in acute ischemic stroke: a systemic review and meta-analysis to aid decision making in patients over 80 years of age. J Neurol Neurosurg Psychiatry 2011;82:712-7.
Thromobolysis in very elderly people: controlled comparison of SITS Internattional Stroke Thrombolysis Registry and Virtual International Stroke Trials Archive. BMJ 2010;341:c6046.
29. Christou I, Alexandrov AV, Burgin WS, et al.Timing of recanalization after tissue plasminogen activator therapy determined by transcranial Doppler correlates with clinical recovery from ischemic stroke. Stroke 2000;31:1812-6. 